\title{
Analysis of organic multilayered samples for optoelectronic devices by (low-energy) dynamic SIMS
}

\author{
K. Q. Ngo, ${ }^{\text {a* }}$ P. Philipp, ${ }^{a}$ Y. Jin, ${ }^{\text {b }}$ S. E. Morris, ${ }^{c}$ M. Shtein, ${ }^{b}$ J. Kieffer $^{\mathrm{b}}$ \\ and T. Wirtz ${ }^{a}$
}

The increasing sophistication of optoelectronic devices requires molecular-level dimensional control in the fabrication of multilayered structures with specifically engineered interfaces. However, the effectiveness of growth and doping strategies devised to achieve the desired device structures often remains unverified due to the lack of adequate characterization techniques. This is particularly true for devices based on conjugated organic compounds, which find increasing use in energy applications (e.g. organic light emitting diodes and organic photovoltaic cells, etc.). The buried interfaces are simply inaccessible or suffer damage when using conventional characterization techniques. In a current project, we address this challenge by advancing the development of low-energy (LE) SIMS for the analysis of organic-based optoelectronic materials systems.

In the present study, multilayered organic thin films have been analyzed, varying experimental conditions such as the impact energies for $\mathrm{Cs}^{+}$bombardment in the $\mathrm{MCs}_{\mathbf{x}}{ }^{+}$and $\mathrm{M}^{-}$mode on a Cameca Sc-Ultra instrument to investigate the ionization mechanisms as well as the atomic mixing at the interfaces between layers, and the degradation of the organic information. Low-energy dynamic SIMS proved to be a reliable tool for the characterization of organic multilayered optoelectronic devices: $\mathrm{MCs}_{x}{ }^{+}$secondary ions provide information about the distribution of elements within the samples, while negative fragments that are characteristic for the different molecules give information about the structure. Copyright (c) 2010 John Wiley \& Sons, Ltd.

Keywords: SIMS; sputtering; low impact energy; depth profile; organic multilayer

\section{Introduction}

In recent years, organic optoelectronic devices (e.g. organic light emitting diodes and organic photovoltaic cells) have known an increasing interest because of their low fabrication costs. ${ }^{[1]}$ With the increasing sophistication of the devices, a meticulous dimensional control of the layered structures is required. However, due to a lack of adequate characterization tools the influence of growth parameters on the structure often remains largely unverified. In a current project, we use low-energy (LE) SIMS to enhance our knowledge on the interfacial structures and electronic properties of such devices. When analyzing such samples by depth profiling in dynamic SIMS, the characterization of the layered structure and the depth resolution are of major importance.

In this paper, we study a multilayer sample composed of three thin films, $\mathrm{C}_{60}$, copper phthalocyanine (CuPC) and bathocuproine $(B C P)$, under low-energy bombardment. Low-impact energies should minimize the fragmentation of the organic molecules, and by conserving the molecular information to a maximum extent, facilitate the identification of the different layers. ${ }^{[2]}$ Furthermore, the atomic mixing due to the collision cascades is reduced. ${ }^{[3]}$ The emission of positive and negative secondary atomic and cluster ions through the different layers is studied as a function of the impact energy.

The fragmentation of $\mathrm{C}_{60}$ and CuPC have also been studied as a function of the impact energy by looking at the abundance distributions of $\mathrm{C}_{n}{ }^{-}, \mathrm{C}_{n} \mathrm{Cs}^{-}$and $\mathrm{C}_{n} \mathrm{~N}_{m}{ }^{-}$cluster ions in the negative secondary ion mode. These results are reported in a separate paper. ${ }^{[4]}$ Here, these distributions are used to select characteristic secondary ions of each molecule and facilitate the interpretation of the depth profile in the negative mode. Complementary information on the sample is obtained from depth profiles in the positive mode, which have been carried out using mainly $\mathrm{MCs}_{\mathrm{x}}+$ ions.

\section{Experimental}

The multilayer sample used in this study consists of three thin films deposited onto glass: $C_{60}(40 \mathrm{~nm}$, top layer), copper phthalocyanine (CuPC, $\mathrm{C}_{32} \mathrm{H}_{16} \mathrm{~N}_{8} \mathrm{Cu}, 25 \mathrm{~nm}$ ) and bathocuproine $\left(\mathrm{BCP}, \mathrm{C}_{26} \mathrm{H}_{20} \mathrm{~N}_{2}, 8 \mathrm{~nm}\right) . \mathrm{C}_{60}$ contains only carbon atoms, while CuPC is a complex with a copper atom in the centre of the phthalocyanine. BCP is the smallest molecule. It contains only two nitrogen atoms per molecule, which accounts for the 4 atomic pecentage of nitrogen in this molecule compared to $14 \%$ for

* Correspondence to: K. Q. Ngo, Department "Science and Analysis of Materials" (SAM), Centre de Recherche Public-Gabriel Lippmann, 41 rue du Brill, L-4422 Belvaux, Luxembourg.E-mail:ngo@lippmann.lu

a Department "Science and Analysis of Materials" (SAM), Centre de Recherche Public-Gabriel Lippmann, 41 rue du Brill, L-4422 Belvaux, Luxembourg

b Department of Materials Science and Engineering, University of Michigan, Ann Arbor, Michigan 48109-2136, USA

c Department of Chemical Engineering, University of Michigan, Ann Arbor, Michigan 48109-2136, USA 
CuPC. The samples have been prepared by using conventional vacuum thermal evaporation. The thin films were deposited at $10^{-6}$ torr pressure onto substrates at room temperature. During the deposition process, the film thickness was controlled using a precalibrated quartz balance.

The depth profiles have been measured using a $\mathrm{Cs}^{+}$primary ion beam on the Cameca SC-Ultra. ${ }^{[5]}$ The same sample was analyzed with different impact energies, i.e. $250 \mathrm{eV}$ (sample voltage $2 \mathrm{kV}$ ), $560 \mathrm{eV}$ (sample voltage $2 \mathrm{kV}$ in negative mode, and $3 \mathrm{kV}$ in positive mode) and $1 \mathrm{keV}$ (sample voltage $5 \mathrm{kV}$ ), and two secondary ion modes. The primary ion currents ranged between 9 and $23 \mathrm{nA}$. The mass resolution $\frac{M}{\Delta M}$ was set to 400 . A contrast aperture of $300 \mu \mathrm{m}$ was used, and the energy slit was closed to a width of $45 \mathrm{eV}$. Depending on the analysis conditions, the primary ion beam was raster-scanned over an area of $500 \mu \mathrm{m}^{2} \times 500 \mu \mathrm{m}^{2}$ (field aperture of 1200), or $300 \mu \mathrm{m}^{2} \times 300 \mu \mathrm{m}^{2}$ (field aperture of 1200 and 750 ), or $250 \mu \mathrm{m}^{2} \times 250 \mu \mathrm{m}^{2}$ (field aperture of 1200). The electron gun was used for charge compensation.

\section{Results and Discussion}

\section{Negative secondary ion mode}

For the negative secondary ion mode, we selected characteristic fragments leading to high secondary ion yields ${ }^{[4]}$ as well as atomic ions of interest, i.e. $\mathrm{O}^{-}, \mathrm{Si}^{-}, \mathrm{C}_{n}{ }^{-}, \mathrm{C}_{n} \mathrm{Cs}^{-}, \mathrm{C}_{n} \mathrm{~N}_{m}{ }^{-}, \mathrm{CuN}_{m}{ }^{-}, \mathrm{CuC}_{n} \mathrm{~N}_{m}{ }^{-}$. On the graphs (Figs 1 and 2), only some secondary ion intensities are shown.

The profile of the top $C_{60}$ layer is characterized by $a$ transient regime followed by steady-state conditions. Variations of secondary ion intensities in this transient regime depend on cluster size and cluster composition. For $\mathrm{C}_{n}{ }^{-}$clusters sputtered from graphite, the intensity variations prior to steady-state have been related to an increase of Cs surface concentration. ${ }^{[6,7]}$ For $\mathrm{C}_{n} \mathrm{Cs}^{-}$clusters, this behaviour is even more pronounced (Fig. 2). The second layer (CUPC) is easily identified based on the intensity increase of the nitrogen containing clusters by several orders of magnitude (Fig. 1). For the different impact energies, clusters containing the different species of the molecule $\left(\mathrm{C}_{n} \mathrm{~N}_{m}{ }^{-}, \mathrm{CuN}_{m}{ }^{-}\right.$, $\left.\mathrm{CuC}_{n} \mathrm{~N}_{m}{ }^{-}\right)$as well as combinations with $\mathrm{Cs}\left(\mathrm{C}_{n} \mathrm{~N}_{m} \mathrm{Cs}^{-}\right)$are detected. In the third layer (BCP), all secondary ion intensities except $\mathrm{O}^{-}$are smaller than in the previous layers.

The different layers can be clearly identified for all impact energies. The $\mathrm{C}_{2} \mathrm{~N}_{2} \mathrm{Cs}^{-}$intensity of the CuPC layer is about two orders of magnitude higher than those of the other layers. The high intensity in the $\mathrm{C}_{60}$ layer can be explained by mass interferences. These observations are also valid for the $\mathrm{Cu}$ containing clusters, although the intensity differences between the layers are somewhat smaller. Furthermore, the sputter rate ratios between the different layers must change with the impact energy. For the $250 \mathrm{eV}$ impact energy, the BCP layer seems to be much thicker compared to others, even though all depth profiles were obtained on the same sample.

\section{Positive secondary ion mode}

In the positive secondary ion mode, the same impact energies as for the negative mode were used. Mainly $\mathrm{MCs}^{+}$and $\mathrm{MCs}_{2}{ }^{+}$cluster intensities were recorded. They are shown in Fig. 3, normalized with respect to the $\mathrm{Cs}^{+}$and $\mathrm{Cs}_{2}{ }^{+}$signals, respectively. The $\mathrm{C}_{60}$ and $\mathrm{CuPC}$ layers produce the highest $\mathrm{CCs}^{+}$and $\mathrm{CCs}_{2}{ }^{+}$intensities. The BCP layer is oxidized, which agrees with the data obtained in
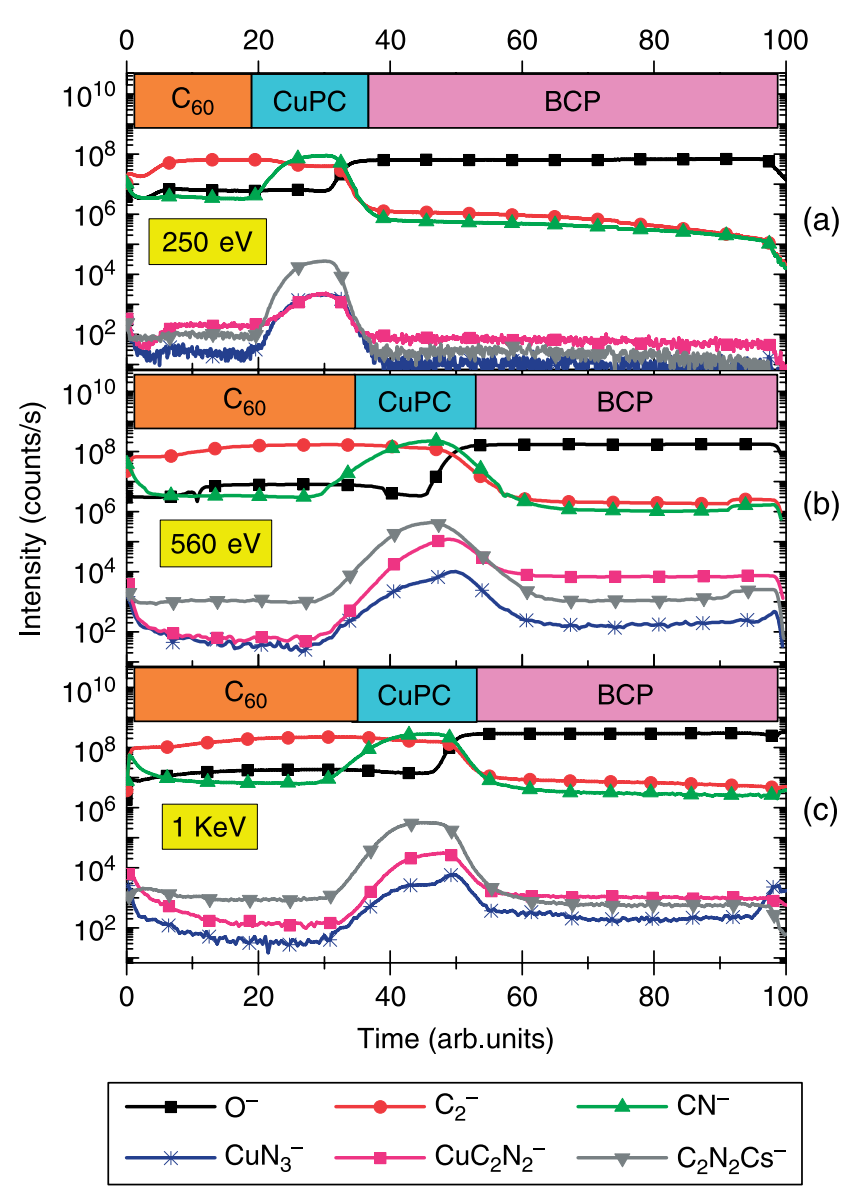

Figure 1. Depth profiles of the C60/CuPC/BCP multilayered sample in the negative mode at, a) $250 \mathrm{eV}$, b) $560 \mathrm{eV}$, and c) $1 \mathrm{keV}$.

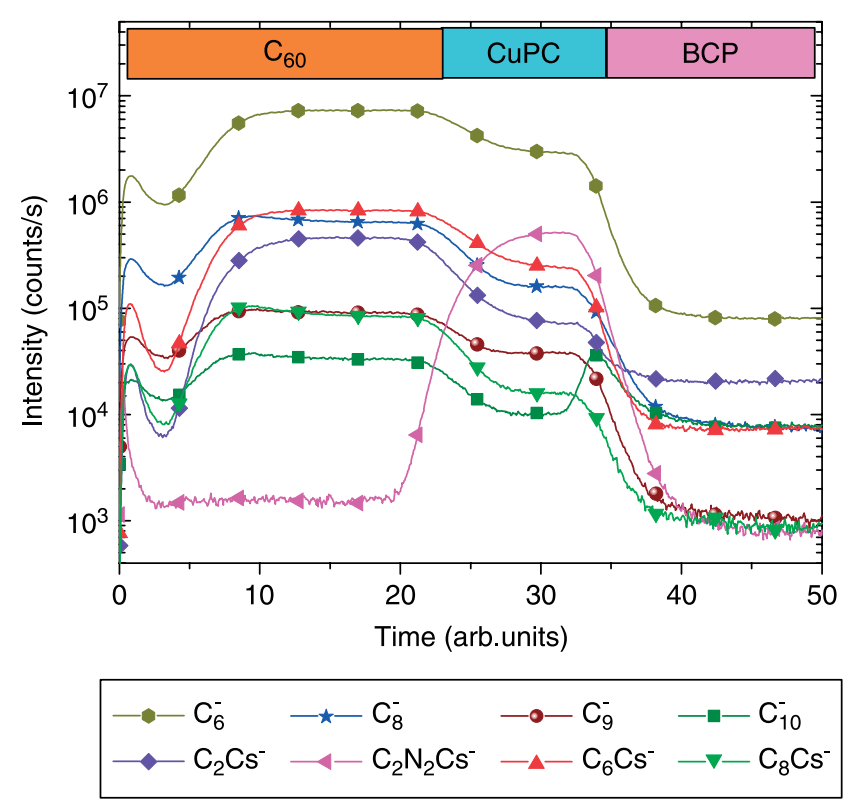

Figure 2. Depth profile at $250 \mathrm{eV}$ for $\mathrm{Cn}^{-}$and $\mathrm{CnCs}^{-}$cluster. 

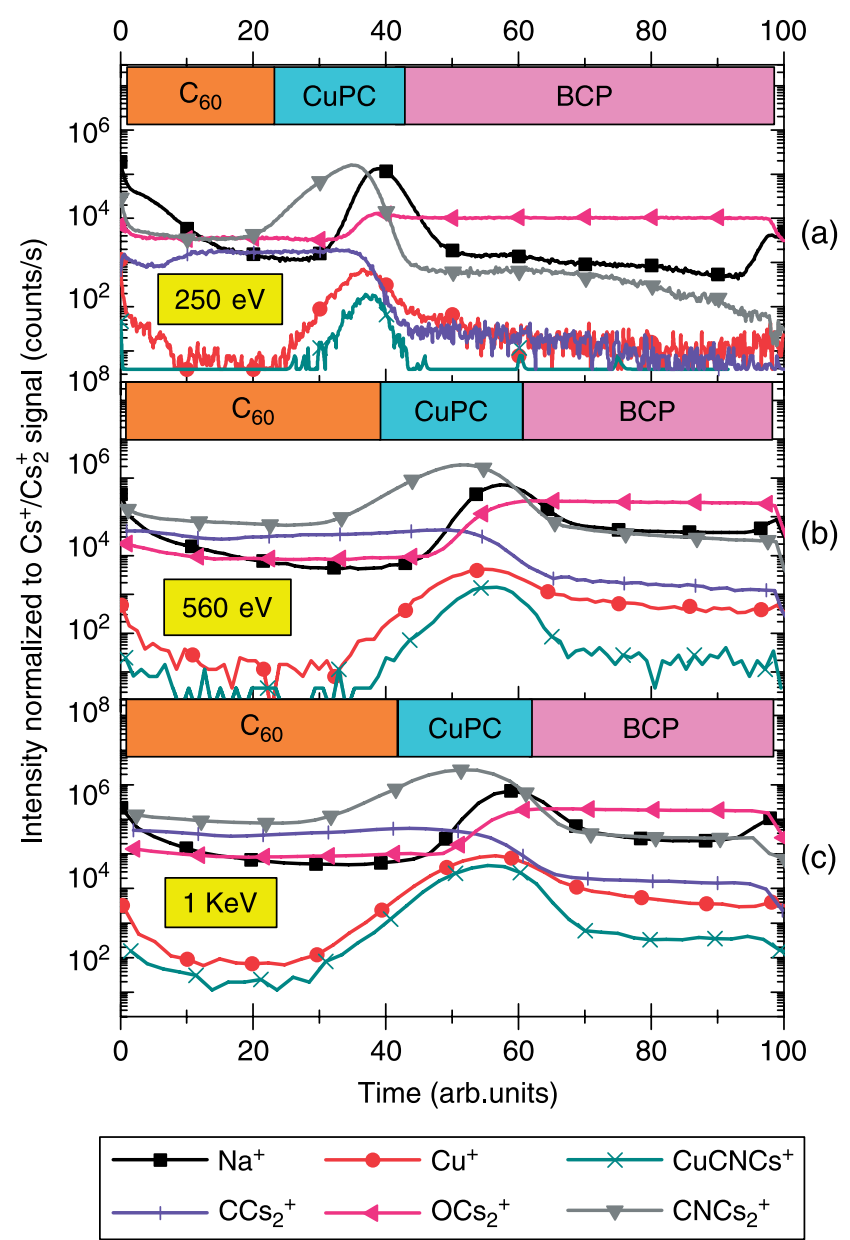

Figure 3. Depth profiles of the $\mathrm{C} 60 / \mathrm{CuPC} / \mathrm{BCP}$ multilayered sample in positive mode at, a) $250 \mathrm{eV}$, b) $560 \mathrm{eV}$, and c) $1 \mathrm{keV}$ impact energies. These intensities have been normalized with respect to $\mathrm{Cs}+$ and $\mathrm{Cs} 2+$ intensities.

the negative mode. The CuPC layer is characterized by an increase of secondary ions containing $\mathrm{Cu}$ or $\mathrm{N}$. Similar to the negative mode, $\mathrm{N}$-containing ions have higher intensities in the CuPC layer than in the BCP layer. Even large clusters like $\mathrm{CuCNCs}^{+}$have been observed although their intensities are much lower than those of smaller clusters $\left(\mathrm{CNCs}_{2}{ }^{+}\right)$. Furthermore, the depth profiles in positive mode allowed identification of a $\mathrm{Na}$ contamination at the interface between the CUPC and BCP layers.

\section{Results and Discussion}

Many studies on the use of low-energy SIMS investigating the atomic mixing and the control of roughness formation have been carried out. ${ }^{[8]}$ Besides, the fragmentation of organic molecules under ion bombardment has been studied for a long time with static SIMS. ${ }^{[9]}$ In this paper, dynamic low-energy SIMS is used for the characterization of layered organic samples. For both the positive and the negative modes, the depth profiles show constant secondary ion intensities in each layer. They have not only been obtained for small fragments or atomic ions, but also for relatively large clusters such as $\mathrm{C}_{2} \mathrm{~N}_{2} \mathrm{Cs}^{-}, \mathrm{C}_{10}{ }^{-}, \mathrm{CuC}_{2} \mathrm{~N}_{2}{ }^{-}$, and $\mathrm{C}_{6} \mathrm{~N}_{3}{ }^{-}$in the negative mode, and $\mathrm{CuCNCs}_{2}{ }^{+}, \mathrm{C}_{5} \mathrm{~N}_{2} \mathrm{Cs}^{+}$, and $\mathrm{C}_{4} \mathrm{~N}_{3} \mathrm{Cs}^{+}$in the positive mode (the last two species in each group are not shown in the graph). These observations indicate that the different organic molecules $\left(\mathrm{C}_{60}, \mathrm{CuPC}\right.$ and $\left.\mathrm{BCP}\right)$ are not completely fragmented by the $\mathrm{Cs}^{+}$bombardment and that sufficient molecular information is conserved for an unambiguous identification of the different layers. Even intensities of large clusters are high enough to be of interest in SIMS.

In contrast to the molecular information, ionization processes are less straightforward for these kinds of molecules, and have not been studied in this project yet. At $250 \mathrm{eV}$ the $\mathrm{C}_{n}{ }^{-}$and $\mathrm{C}_{n} \mathrm{Cs}^{-}$ intensities decrease between the $C_{60}$ and CuPC layer, while no variation of these intensities is observed at higher-impact energies. This is probably related to the variation of the Cs concentration. It has been shown that the electron tunneling model can be applied to graphite ${ }^{[6,7]}$ and amorphous carbon. ${ }^{[10]}$ To what extend this model can be applied to the organic semiconductor materials used in this study remains to be verified. Comparison between the graphs is complicated by the change of the mass spectrometer transmission (sample voltage of $\pm 2 \mathrm{kV}$ for $250 \mathrm{eV},-2 \mathrm{kV}$ and $-3 \mathrm{kV}$ for $560 \mathrm{eV}$, and $\pm 5 \mathrm{kV}$ for $1 \mathrm{keV}$ ).

All depth profiles in Figs 1 and 3 extend to the interface with the glass substrate. For both secondary ion modes, the apparent thickness of the layers seems to change with the impact energy, especially for the BCP layer. The latter seems to be much thicker when probed with $250 \mathrm{eV}$ primary ions. Actually, the first layer, $C_{60}$, is the thickest $(40 \mathrm{~nm})$, followed by CuPC $(25 \mathrm{~nm})$ and BCP $(8 \mathrm{~nm})$. Hence, for a given energy, the relative layer thicknesses revealed by the depth profiles do not correspondent to the real thicknesses, indicating that the sputter rates of the different layers must be significantly different. Those changes are probably related to the crystallization of the BCP layer. Some studies proved that BCP is crystalizing just after few hours in air. ${ }^{[11]}$ In our study, the presence of oxygen reveals the crystallization of the BCP layer, both in the negative and positive secondary ion mode (Figs 1 and 2).

The sputter rate of different layers has been calculated separately for $\mathrm{C}_{60}$ and CuPC. At $560 \mathrm{eV}$ and a raster size of $300 \mu \mathrm{m}^{2} \times 300 \mu \mathrm{m}^{2}$, the sputter rate is $0.03 \mathrm{~nm} / \mathrm{s}\left(I_{p}=20 \mathrm{nA}\right)$ for $\mathrm{C}_{60}$ and $0.1 \mathrm{~nm} / \mathrm{s}\left(I_{p}=20 \mathrm{nA}\right)$ for CuPC. For the same impact energy and with a raster size of $250 \mu \mathrm{m}^{2} \times 250 \mu \mathrm{m}^{2}$, the sputter rate of $\mathrm{Si}$ is $0.34 \mathrm{~nm} / \mathrm{s}\left(\mathrm{I}_{p}=30 \mathrm{nA}\right)$. The change in sputter rates between $\mathrm{C}_{60}$ and CuPC is due to different molecular structures. For similar conditions, the CuPC and Si sputter rates differ only slightly. The value of BCP is not calculated as the crystalization modified the layer characteristics.

The depth resolution in the different profiles is not very high. Depending on the impact energy and the secondary ion mode, the interface appears sharper, but this is mostly due to the distortion of the layer thicknesses. The relatively low depth resolution is probably due to layer interdiffusion during sample growth. Nevertheless, the peak of the Na contamination is better resolved for a low-impact energy.

\section{Conclusion}

Traditionally, inorganic samples have been analyzed using dynamic SIMS while static SIMS was used for organic samples. In this paper, we present first low-energy dynamic SIMS results for an organic multilayered sample for optoelectronic devices. The impact energy was changed between $1 \mathrm{keV}$ and $250 \mathrm{eV}$ for both positive and negative secondary ion modes. For the negative mode, the fragmentation analysis of the organic molecules is reported in another paper. In this paper, characteristic fragments of the different molecules were selected to study the variation of 
secondary ion intensities with respect to the impact energy and the layer composition as well as the behavior of the different layers under ion bombardment. Our results, concerning the interface structure, contaminations and the oxidation of layers are of particular interest for the fabrication of organic optoelectronic devices.

The three thin layers $\mathrm{C}_{60} / \mathrm{CuPC} / \mathrm{BCP}$ are well separated in the low-energy SIMS depth profiles. Constant secondary ion intensities (even for large clusters) confirm that enough molecular information is conserved for the unambiguous identification of the different layers. The $C_{60}$ layer has higher intensities of $C_{n}{ }^{-}$ and $\mathrm{C}_{n} \mathrm{Cs}^{-}$clusters than the other layers at $250 \mathrm{eV}$. No difference was observed between the $\mathrm{C}_{60}$ and CuPC layers at the higherimpact energies. The CuPC layer was easy to recognize through the increased intensity of clusters containing nitrogen or copper, such as $\mathrm{CN}^{-}, \mathrm{CuN}_{3}{ }^{-}, \mathrm{CuC}_{2} \mathrm{~N}_{2}^{-}, \mathrm{C}_{2} \mathrm{~N}_{2} \mathrm{Cs}^{-}$, etc. in the negative mode, and $\mathrm{Cu}^{+}, \mathrm{CuCNCs}^{+}, \mathrm{CNCs}_{2}{ }^{+}$, etc. in the positive mode. The depth profiles revealed that the layer of $\mathrm{BCP}$ is oxidized, while some $\mathrm{Na}$ contamination has been detected in between the CUPC and BCP layers.

The influence of surface roughness formation under ion bombardment as well as the initial roughness of the layers on the depth resolution has not been studied in this paper and will be included in future work.

\section{References}

[1] S. E. Shaheen, D. S. Ginley, G. E. Jabbour, MRS bulletin 2005, 30, 10.

[2] H. G. Cramer, T. Grehl, F. Kollmer, R. Moellers, E. Niehuis, D. Rading, Appl. Surf. Sci. 2008, 255, 966.

[3] A. R. Chanbasha, A. T. S. Wee, J. Vac. Sci. Technol. B 2006, 24, 547.

[4] K. Q. Ngo, P. Philipp, M. Shtein, J. Kieffer, T. Wirtz, Surf. Interface Anal. 2009.

[5] E. D. Chambost, B. Boyer, B. Rasser, M. Schuhmacher, Proceeding of the twelvelnternational Conference on Secondary Ion Mass Spectrometry (SIMS XII) 1999, 533.

[6] H. Gnaser, Phys. Rev. B 2001, 63, 45415.

[7] H. Gnaser, Appl. Surf. Sci. 2003, 203, 78.

[8] N. Toyoda, J. Matsuo, T. Aoki, I. Yamada, D. B. Fenner, Nucl. Instrum. Methods Phys. Res. B 2002, 190, 860.

[9] A. Delcorte, P. Bertrand, J. C. Vickerman, B. J. Garrison, Proceeding of the twelvelnternational Conference on Secondary Ion Mass Spectrometry (SIMS XII) 1999, 27.

[10] A. K. Gupta, P. Ayyub, Eur. Phys. J. D 2001, 17, 221.

[11] H. R. Wu, Q. L. Song, M. L. Wang, F. Y. Li, H. Yang, Y. Wu, C. H. Huang, X. M. Ding, X. Y. Hou, Thin Solid Films 2007, 515, 8050. 